\title{
Trefoil Factor 3 is Highly Predictive of Complete Mucosal Healing Independently and in Combination with C-Reactive Protein in Patients with Ulcerative Colitis
}

\author{
Radislav Nakov ${ }^{1}$, Tsvetelina Velikova ${ }^{2}$, Ventsislav Nakov ${ }^{1}$, Vanya Gerova ${ }^{1}$, Lyudmila Tankova ${ }^{1}$
}

1) Clinic of Gastroenterology,

Tsaritsa Yoanna University

Hospital, Medical University

of Sofia, Sofia

2) Laboratory of Clinical Immunology, University Hospital Lozenetz, Sofia, Bulgaria

\author{
Address for correspondence: \\ Radislav Nakov \\ 8 Byalo More Str., \\ Clinic of Gastroenterology \\ Tsaritsa Yoanna University \\ Hospital, \\ 1527 Sofia, Bulgaria \\ radislav.nakov@gmail.com
}

Received: 19.02 .2019

Accepted: 05.04.2019

\begin{abstract}
Aim: In the current study we aimed to evaluate the role of trefoil factor 3 (TFF3) as a marker for complete mucosal healing $(\mathrm{MH})$ in patients with ulcerative colitis (UC).

Methods: We enrolled 116 consecutive UC patients. Trefoil factor 3 levels were measured by ELISA and were compared to the clinical activity, assessed by Lichtiger Index, fecal calprotectin (FCP) and C-reactive protein (CRP) levels. Colonoscopy was performed in all the patients and the findings were graded according to Mayo endoscopic score (EMS) and UC endoscopic index of severity (UCEIS).

Results: Trefoil factor 3 levels were significantly correlated with Lichtiger Index $(r=0.736, p<0.001)$, EMS $(\mathrm{r}=0.811, \mathrm{p}<0.001)$, UCEIS $(\mathrm{r}=0.820, \mathrm{p}<0.001)$, FCP $(\mathrm{r}=0.696, \mathrm{p}<0.001)$ and CRP $(\mathrm{r}=0.405, \mathrm{p}<0.001)$. The TFF3 cut-off level of $6.74 \mathrm{ng} / \mathrm{ml}$ indicated complete $\mathrm{MH}$ (EMS $=0$; UCEIS $=0$ ) with a sensitivity and specificity of 0.879 and 0.869 , respectively (area under the curve, AUC 0.927; 95\% confidence interval, 0.877-0.976). The DeLong's test revealed no significant difference between the AUC of TFF3+CRP and the AUC of FCP $(\mathrm{Z}=1.717, \mathrm{p}=0.086)$, AUC of TFF3+FCP $(\mathrm{Z}=1.908, \mathrm{p}=0.056)$, and AUC of TFF3+CRP+FCPP $(\mathrm{Z}=1.915$, $\mathrm{p}=0.056$ ). However, the AUC of TFF3+CRP showed significant difference compared with the AUC of TFF3 $(\mathrm{Z}=2.210, \mathrm{p}=0.027)$ and the AUC of CRP $(\mathrm{Z}=3.145, \mathrm{p}=0.002)$ for predicting complete $\mathrm{MH}$.

Conclusions: Trefoil factor 3 levels correlated significantly with clinical activity, endoscopic indices, CRP and FCP in our patients. TFF3 is a highly predictive marker of complete MH independently and in combination with CRP in patients with UC.
\end{abstract}

Key words: inflammatory bowel diseases - biomarkers - fecal calprotectin - trefoil factor 3.

Abbreviations: CRP: C-Reactive protein; FCP: fecal calprotectin; IBD: inflammatory bowel disease; MH: mucosal healing; TFF3: trefoil factor 3; UC: ulcerative colitis; UCEIS: ulcerative colitis endoscopic index of severity.

\section{INTRODUCTION}

Ulcerative colitis (UC) is a lifelong chronic inflammatory bowel disease (I B D ) characterized by periods of remission and relapse [1]. It is currently defined by a continuous mucosal inflammation of the rectum and a variable extent of the colon, without granulomas on mucosal biopsies [1].

In the past, clinical remission was the main therapeutic goal for patients with UC and the disease management aimed to control the symptoms, such as rectal bleeding and increased frequency of bowel movements [2]. However, there is mounting evidence that achieving clinical remission without mucosal healing $(\mathrm{MH})$ is not associated with reduced rates of hospitalization or colectomy over the years [3]. Hence, MH has become the treatment target in UC patients, leading to improvement of short- and long-term outcomes [4-6].

The use of repeated endoscopy is the most accurate way to evaluate $\mathrm{MH}$, however this is invasive, time-consuming, and expensive [7]. Therefore, identification of noninvasive markers to accurately detect mucosal inflammation is needed. To date, the best studied inflammatory markers are $\mathrm{C}$-reactive protein (CRP) and fecal calprotectin (FCP). Despite the reported correlation between endoscopic activity and CRP [8], data are still insufficient to warrant its broad use in UC. There are many promising results for FCP $[9,10]$, but more studies are needed to clarify adequate surveillance strategies and cut-off 
levels before its broad implementation in clinical practice. Consequently, there is still need for new markers to identify intestinal inflammation in IBD.

Trefoil factors (TFFs) include a family of three mucinassociated peptides secreted by goblet cells in the intestinal mucosa [11]. They have a key role in maintaining mucosal barrier integrity [12] and are up-regulated at the site of mucosal damage $[13,14]$. It has recently been suggested that serum levels of TFF3 can predict disease activity [15] and reflect $\mathrm{MH}$ in UC patients with minimal disease activity [16]. However, its potential to predict complete $\mathrm{MH}$ independently or in combination with other biomarkers has never been addressed.

In the current study, we aimed to evaluate the diagnostic role of TFF3 as a noninvasive marker for complete mucosal healing in patients with UC and to compare its levels with those of FCP and CRP.

\section{METHODS}

This was a prospective observational study. We prospectively recruited patients referred to the IBD outpatient clinic of "Tsaritsa Yoanna" University Hospital in Sofia between October 2015 and February 2018. As inclusion criteria we used: age 18-85 years, known UC diagnosed according to the ECCO Guidelines [1] and completion of a written informed consent. The exclusion criteria were: colorectal cancer or colon polyps, indeterminate colitis, history of colorectal surgery, pregnancy, history of active non-steroidal anti-inflammatory drugs (NSAIDs) intake (2 tablets/week), oral steroids or steroid enemas intake in the last 3 months, infectious colitis and primary immunodeficiency.

We defined clinical remission in UC as a Lichtiger Clinical Activity Index of 3 points or less [17] and endoscopic remission as Ulcerative Colitis Endoscopic Index of Severity (UCEIS) of 0 [18]. As a complete MH we defined UCEIS of 0 and EMS of 0 .

All enrolled patients underwent a full medical assessment including a detailed medical history and physical examination. All therapies received by the patients before enrollment were recorded. All patients underwent colonoscopy within two weeks from the visit. Serum and fecal samples were collected within 1-2 days before colonoscopy. Serum levels of TFF3, CRP and FCP levels were assessed in all the patients.

\section{Enzyme immunoassay}

Serum levels of TFF3 were measured by ELISA (Quantikine ELISA, Human TFF3 Immunoassay, R\&D Systems, Inc., USA). We strictly followed the manufacturer's instructions.

\section{Fecal calprotectin}

Calprotectin was analyzed in stool samples by means of point-of-care desk-top Quantum Blue Reader ${ }^{\circledast}$ (POC Reader) method. It is a lateral flow technology based on ELISA techniques. We performed the test according to the manufacturer's instructions (Quantum Blue ${ }^{\varpi}$ Calprotectin, Bühlmann Laboratories AG, Switzerland) [19]. The POC device uses internal standards within a range of 30-300 $\mu \mathrm{g} / \mathrm{g}$ and a sensitivity of $<10 \mu \mathrm{g} / \mathrm{g}$, thus, guarantying consistency in results. When we received results $>300 \mu \mathrm{g} / \mathrm{g}$, we performed additional 1:10 dilution with extraction buffer according to the manufacturer's instructions, allowing us to receive FCP levels up to $3000 \mu \mathrm{g} / \mathrm{g}$. FCP values above the upper limit of the measurement ranges were registered as $3000 \mu \mathrm{g} / \mathrm{g}$ and FCP values below the lower limit were accordingly registered as $30 \mu \mathrm{g} / \mathrm{g}$.

\section{Statistical analysis}

The statistical analysis was performed using SPSS for Windows, Version 25.0. (SPSS Inc., Chicago, USA) and MedCalc Statistical Software, Version 16.4.3 (MedCalc Software bvba, Ostend, Belgium; 2016). Descriptive statistic for tabular and graphical presentation of results was used. The inter-rater agreement of the endoscopic indices was analyzed using Cohen's weighted Kappa coefficient. Correlation analysis was performed using Spearman's and Pearson's correlation coefficient. The comparison between the coefficients of correlations was conducted using Steiger's Z test, known also as Meng's test [20]. The receiver operating characteristic (ROC) curves for the TFF3, CRP and FCP levels were assessed to predict the complete $\mathrm{MH}$. The comparison between the area under the ROC curve was analyzed using DeLong's test [21]. A p value of $<0.05$ was considered statistically significant.

\section{Ethics approval}

The current study was approved by the Ethics Committee of “Tsaritsa Yoanna” University Hospital in Sofia, Bulgaria. Before initiating this study, written informed consent was obtained from all patients. The study protocol conforms to the ethical guidelines of the 1975 Declaration of Helsinki (6th revision, 2008) as reflected in a priori approval by the institution's human research committee.

\section{RESULTS}

The current study enrolled 116 UC patients, 62 (53.4\%) were men; the median age at diagnosis of UC was of $39.4 \pm$ 9 (19-65) years. The proportion of proctitis, left-sided colitis and extensive colitis in the cohort was $15.5 \%, 43.1 \%$, and $41.4 \%$, respectively. Fifty-six $(48.3 \%)$ of the patients were with quiescent disease and 60 (51.7\%) with active UC. An overview of the demographic patient characteristics at baseline is provided in Table I.

The mean FCP level was $926.28 \mu \mathrm{g} / \mathrm{g}$ (30-3000; SD 1101.72), the mean CRP level was $2.11 \mathrm{mg} / \mathrm{dl}$ (0.02-28.62; SD 4.69) and the mean TFF3 level was $8.03 \mathrm{ng} / \mathrm{ml}$ (4.6-13.54; SD 2.76), whereas the median EMS, UCEIS and Lichtiger Index values were 1.25 (0-3; SD 1.31), 2.13 (0-7; SD 2.25), and 4.72 (0-17; SD 4.75), respectively (Table II). Fifty patients (43\%) were with complete $\mathrm{MH}$ and 66 (57\%) with active UC. The mean levels of the three biomarkers, clinical and endoscopic indices are provided in Table II.

\section{Correlation of the TFF3 level with FCP and CRP levels}

Trefoil factor 3 levels exhibited significant correlation with FCP levels (Pearson correlation coefficient $r=0.696, p<0.001$ ) and CRP levels ( $\mathrm{r}=0.405, \mathrm{p}<0.001)$ (Figs. 1D, 1E). Moreover, FCP levels correlated significantly with CRP levels $(r=0.451, p$ $<0.001$ ) (Fig. 1F). When comparing the degree of correlation between the TFF3 levels and other biomarkers, FCP showed 
Table I. Characteristics of Ulcerative Colitis Patients Enrolled in the Study

\begin{tabular}{lcc}
\hline Characteristics & Number & $\begin{array}{c}\text { Percentage } \\
(\%)\end{array}$ \\
\hline No. patients & 116 & - \\
Gender & & \\
Male & 62 & 53.4 \\
Female & 54 & 46.6 \\
Age, mean \pm SD, range (years) & $39.4 \pm 9(19-65)$ & - \\
Duration of the disease, mean \pm SD & $4.1 \pm 2.8$ & - \\
(years) & & \\
Smoking habit & & \\
Smokers & 32 & 27.6 \\
Never smoked & 70 & 60.3 \\
Ex-smokers & 14 & 12.1 \\
Disease location & & \\
Proctitis - E1 & 18 & 15.5 \\
Left-sided colitis - E2 & 50 & 43.1 \\
Extensive colitis - E3 & 48 & 41.4 \\
Therapy & & \\
None & 7 & 6.0 \\
Topical 5-ASA & 17 & 14.7 \\
Systemic 5-ASA & 58 & 50.0 \\
Azathioprine & 34 & 29.3 \\
TNF-alpha inhibitor & 23 & 19.8 \\
\hline
\end{tabular}

a better correlation with the TFF3 levels, as compared to CRP (Steiger's $\mathrm{Z}$ test $\mathrm{Z}=3.209, \mathrm{p}<0.01$ ).

Correlation of the TFF3 level with the disease activity index and endoscopic indices

Trefoil factor 3 levels exhibited significant correlation with Lichtiger Index (Spearman correlation coefficient $r=0.736$, $\mathrm{p}<0.001)$, EMS $(\mathrm{r}=0.811, \mathrm{p}<0.001)$ and UCEIS $(\mathrm{r}=0.820$, $\mathrm{p}<0.001$ ) (Figs. 1A, 1B and 1C). Furthermore, FCP levels correlated significantly with EMS $(r=0.839, p<0.001)$ and UCEIS $(r=0.872, p<0.001)$. C-reactive protein levels also had significant correlation with EMS $(r=0.610, p<0.001)$ and UCEIS $(\mathrm{r}=0.665, \mathrm{p}<0.001)$. Trefoil factor 3 levels had a significantly better correlation with UCEIS compared to CRP $(\mathrm{z}=-2.911$, $\mathrm{p}<0.01)$ but a similar correlation with UCEIS compared to FCP levels $(\mathrm{Z}=1.707, \mathrm{p}<0.01)$. UCEIS showed very strong correlation with EMS ( $\mathrm{r}=0.934, \mathrm{p}<0.001)$. The kappa coefficient of inter-rater agreement for MES and UCEIS were 0.80 and 0.76 , respectively.

\section{Analysis of the ROC curves}

ROC curve analysis indicated a TFF3 cut-off level of 6.74 $\mathrm{ng} / \mathrm{ml}$ (area under the curve [AUC], 0.927; 95\% CI, 0.863 0.967 ) for predicting complete MH defined as both UCEIS and EMS values of 0 . The sensitivity and specificity of the cut-off value of $6.74 \mathrm{ng} / \mathrm{ml}$ for complete $\mathrm{MH}$ were $87.9 \%$ and $86.9 \%$, respectively (Fig. 2C). ROC analysis revealed CRP cut-off level of $0.43 \mathrm{mg} / \mathrm{dl}$ (AUC, 0.865 ; 95\% CI, 0.789 - 0.921) for predicting complete $\mathrm{MH}$ with sensitivity of $78.8 \%$ and specificity of $76 \%$ (Fig. 2B). ROC curve analysis showed a FCP cut-off level of $99 \mu \mathrm{g} / \mathrm{g}$ (AUC, 0.988; 95\% CI, 0.947 - 0.999) for predicting complete $\mathrm{MH}$. The sensitivity and specificity of this cut-off value for complete $\mathrm{MH}$ were $97 \%$ and $98 \%$, respectively (Fig. 2A).

After combining the biomarkers among them, the AUC of TFF3+CRP was 0.960 (95\% CI, 0.907-0.988), the AUC of TFF3+FCP was 0.990 (95\% CI, 0.951-1.000), and the AUC of TFF3+CRP+FCPP was also 0.990 (95\% CI, 0.951-1.000) (Figs. 3 and 4).

When comparing the AUC of TFF3 with the AUC of CRP and the AUC of FCP for predicting complete $\mathrm{MH}$, the DeLong's test showed lack of significant difference for the former $(\mathrm{Z}=1.543, \mathrm{p}=0.0123)$ and significant difference for the latter $(\mathrm{Z}=2.380, \mathrm{p}=0.017)$ (Fig. 2D). There was a significant difference between the AUC of TFF3 and the AUC of TFF3+CRP $(Z=2.210, p=0.027)$, the AUC of TFF3+FCP $(Z=2.525, p 0.012)$, and AUC of TFF3+CRP+FCPP $(Z=2.482, p=0.013)$.

DeLong's test revealed no significant difference between the AUC of TFF3+CRP and the AUC of FCP $(Z=1.717$, $\mathrm{p}=0.086)$, AUC of TFF3+FCP $(\mathrm{Z}=1.908, \mathrm{p}=0.056)$, and AUC of TFF3+CRP+FCPP $(Z=1.915, p=0.056)$. However, the AUC of TFF3+CRP showed significant difference with the AUC of TFF3 ( $Z=2.210, p=0.027)$ and the AUC of CRP $(Z=3.145$, $\mathrm{p}=0.002)$ for predicting complete $\mathrm{MH}$. The DeLong's test did not find significant difference between the AUC of FCP alone and the AUC of any combination of biomarkers.

\section{DISCUSSION}

It is increasingly believed that achieving $\mathrm{MH}$ may improve quality of life, prevent relapses, minimize hospitalizations, and prevent complications such as colorectal cancer, and

Table II. Mean levels of Lichtiger Index, Mayo endoscopic score (EMS), Ulcerative Colitis endoscopic index of severity (UCEIS), fecal calprotectin (FCP), C-reactive protein (CRP) and trefoil factor 3 (TFF3) in all patients with UC, UC patients with mucosal healing $(\mathrm{MH})$ and active UC patients. All data are presented in mean levels, minimal and maximal values and standard deviation.

\begin{tabular}{|c|c|c|c|}
\hline & All UC patients & UC patients with $\mathrm{MH}$ & Active UC \\
\hline Number & 116 & 50 & 66 \\
\hline Lichtiger Index & $4.72(0-17 ;$ SD 4.75$)$ & $0.64(0-2 ;$ SD 0.77) & 7.82 (0-17; SD 5.86) \\
\hline EMS & $1.25(0-3 ;$ SD 1.31) & 0 & $2.20(0-3 ;$ SD 0.96)) \\
\hline UCEIS & $2.13(0-7 ;$ SD 2.25) & 0 & $3.74(1-7 ;$ SD 1.68) \\
\hline $\mathrm{FCP}(\mu \mathrm{g} / \mathrm{g})$ & 926.28 (30-3000; SD 1101.72) & 50.66 (30-156; SD 26.19) & 1589.62 (51-3000; SD 1053.76) \\
\hline $\mathrm{CRP}(\mathrm{mg} / \mathrm{dl})$ & $2.11(0.02-28.62 ;$ SD 4.69) & $0.29(0.02-1.30 ;$ SD 0.35) & $3.48(0.04-28.62 ;$ SD 5.86) \\
\hline TFF3 (ng/ml) & $8.03(4.6-13.54 ;$ SD 2.76$)$ & $5.79(4.60-10.93 ;$ SD 1.07) & 9.73 (4.69-13.54; SD 2.41) \\
\hline
\end{tabular}




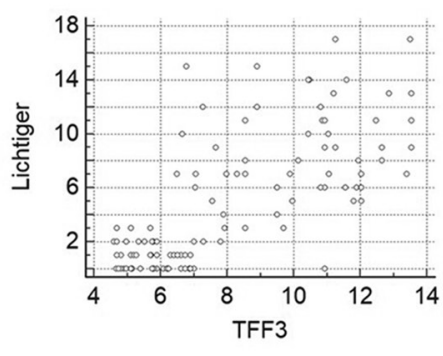

A

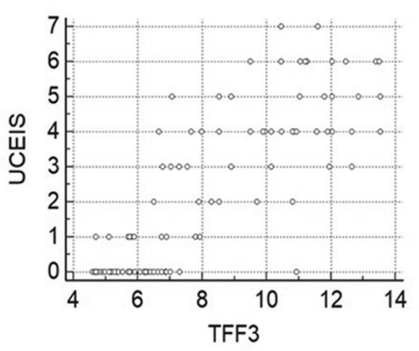

C

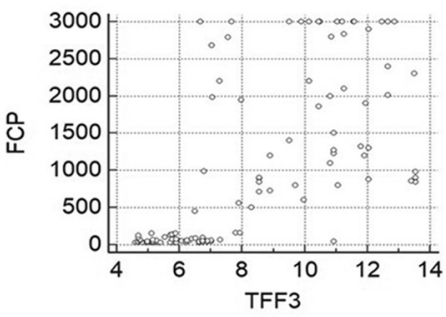

$\mathrm{E}$

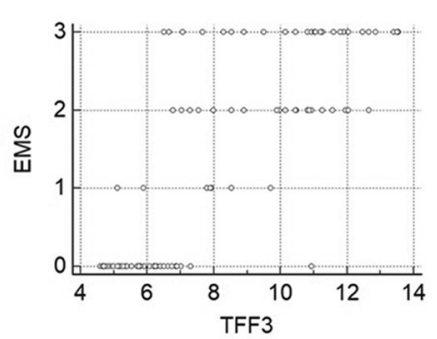

B

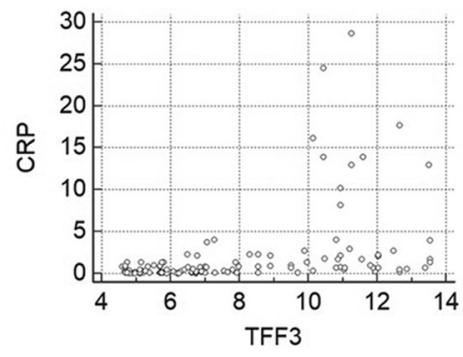

D

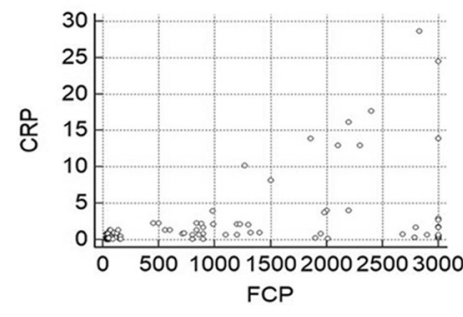

$\mathrm{F}$
Fig. 1. Correlation between trefoil factor levels and A) Lichtiger Clinical Activity Index; B) Mayo endoscopic score (EMS); C) Ulcerative Colitis Endoscopic Index of Severity (UCEIS); D) C-reactive protein (CRP) ; E) Fecal calprotectin (FCP); and correlation between FCP and CRP (F). need for surgery in UC patients [22]. Furthermore, $\mathrm{MH}$ is currently viewed as the best predictor of disease prognosis [2]. However, it is not still clear whether the "gold standard" for $\mathrm{MH}$ assessment, i.e. endoscopy with biopsies, could be replaced by any non-invasive biomarker, more convenient to use in everyday clinical practice. As a factor that plays a pivotal role in maintaining the integrity of intestinal mucosa, TFF3 could be considered as a potential candidate.

According to our best knowledge, the current study is the first that aimed to evaluate the potential of TFF3 to predict complete $\mathrm{MH}$ in UC patients independently or in combination with other biomarkers. Overall, we found that TFF3 levels were highly predictive of complete $\mathrm{MH}$ in UC. Moreover, the combination of TFF3 and CRP was able to predict complete $\mathrm{MH}$ even better than TFF3 alone and with no significant difference when compared to FCP.

Although there are several cellular and animal studies that demonstrated the key role of TFF3 in the integrity maintenance of intestinal mucosa, we are lacking clinical data about its potential in IBD management. Vestergaard et al. [23] did not find any significant fluctuations of TFF3 in three patients with UC who underwent treatment with prednisolone with clinical improvement. Grønbaek et al. [24] found that serum TFF3 levels correlated with disease activity indices in patients with UC and they noted a trend towards reduction in TFF3 levels with clinical improvement after therapy with steroids. In a recent study, Srivastava et al. [16] showed that serum TFF3 could point out patients with $\mathrm{MH}$ in a group of UC patients in clinical remission or with mild activity with reasonable sensitivity and specificity. However, the $\mathrm{MH}$ was defined as Baron score 0 or 1 , and the latter did not represent a complete $\mathrm{MH}$. In one of our recent studies we showed that the mean levels of TFF3 in active UC were significantly higher than those identified in patients with quiescent UC, which were similar to those of the healthy controls [15]. In the current study we demonstrated a strong correlation of TFF3 with clinical activity, endoscopic indices and FCP, showing its potential as a marker for disease activity and complete MH in UC patients. Unfortunately, TFF3 is not a useful marker for disease activity in patients with Crohn's disease $[15,25]$.

Many non-invasive markers have been investigated over years, but still an ideal marker to detect and monitor $\mathrm{MH}$ in UC is needed. Nowadays, FCP is the most studied and the most sensitive one. Based on our ROC curve analysis we could state that FCP significantly predicts complete $\mathrm{MH}$ in UC patients. In spite of the fact that CRP and TFF3 in combination with FCP enhanced the predictability of complete $\mathrm{MH}$, combination 


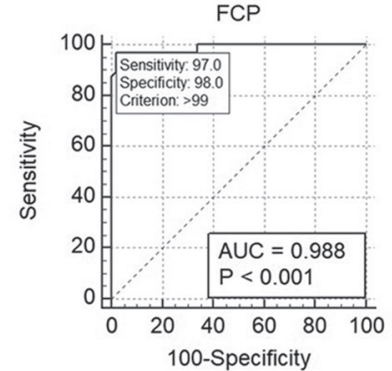

A

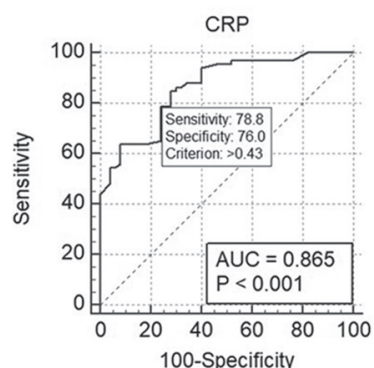

B

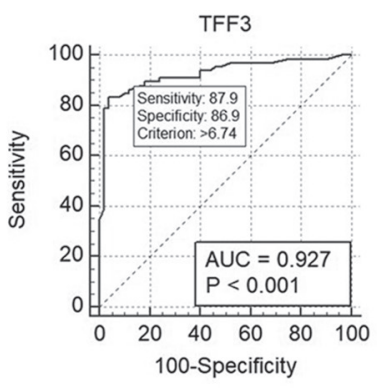

$\mathrm{C}$

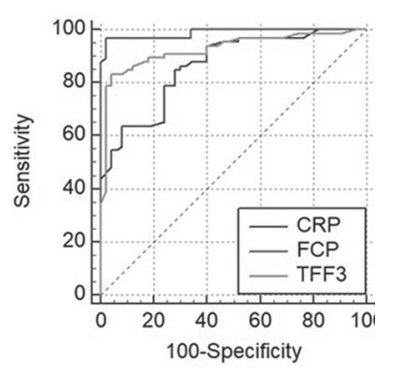

$\mathrm{D}$
Fig. 2. ROC curve analysis showing the AUC of the A) FCP levels; B) CRP levels and C) TFF3 for predicting complete mucosal healing defined as both UCEIS and EMS values of 0 . D) Comparison of the ROC curves of CRP, FCP and TFF3. TFF3 trefoil factor 3, CRP $\mathrm{C}$-reactive protein, FCP fecal calprotectin, $\mathrm{ROC}$ receiver operating characteristics, AUC area under curve, EMS Mayo endoscopic score, UCEIS ulcerative colitis endoscopic index of severity.

of other markers to FCP did not affect significantly the predictability of complete $\mathrm{MH}$.

Although FCP is proved to be the best marker of disease activity in UC, some concerns have been raised. When groups of UC patients with different levels of disease activity are compared, considerable overlaps in FCP levels between the groups were reported [26]. This makes it difficult to interpret the results for the individual patient. A significant day-to-day variation in FCP in patients with UC has also been described [27-29]. Furthermore, there is a lack of validated cut-off level to predict $\mathrm{MH}$ in UC patients. A recent meta-analysis by Rokkas et al. [30] shows best specificity of $78.2 \%$ for cut-off levels of FCP greater than $100 \mu \mathrm{g} / \mathrm{g}$, which is in line with our results.

Interestingly, the combination of TFF3 and CRP has comparable predictability of complete $\mathrm{MH}$ to FCP, which gives an opportunity to UC patients to avoid stool sampling and to be followed-up just by blood tests. This could be extremely useful for patients reluctant to handle fecal material.

\section{CONCLUSIONS}

In the present study we found that serum human TFF3 is significantly correlated with clinical activity, endoscopic indices and FCP in UC patients. Moreover, TFF3 is a highly predictive biomarker of complete $\mathrm{MH}$ independently and in combination with CRP in patients with UC. Future studies on different patient populations are required to further explore

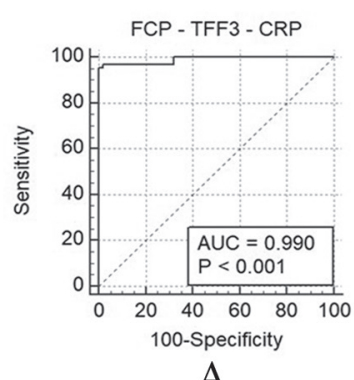

A

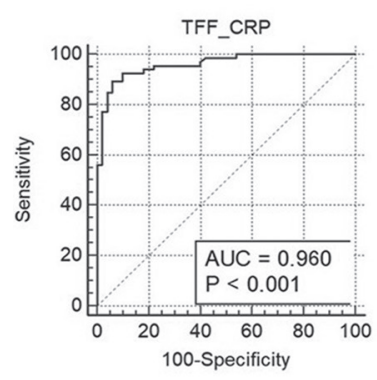

$\mathrm{C}$

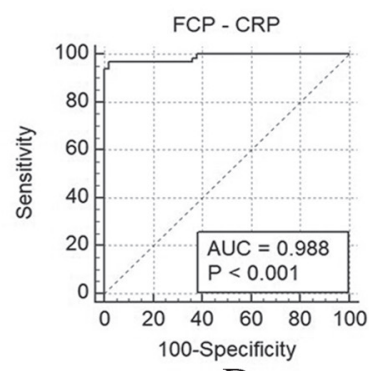

$\mathrm{D}$

Fig. 3. ROC curve analysis showing the AUC of the A) FCP levels in combination with TFF3 and CRP levels (FCP-TFF3-CRP); B) FCP levels in combination with TFF3 levels (FCP-TFF3), C) TFF3 levels in combination with CRP levels (TFF3_CRP) and D) FCP levels in combination with CRP levels (FCP-CRP) for predicting complete mucosal healing defined as both UCEIS and EMS values of 0. (For abbreviations see Fig. 2).

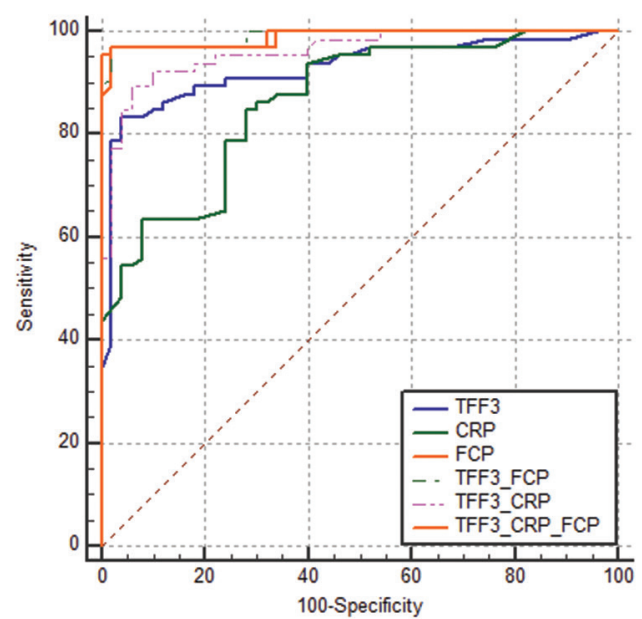

Fig. 4. Comparison of the ROC curves of TFF3, CRP, FCP, combination of TFF3 and FCP levels (TFF3_FCP), combination of TFF3 and CRP levels (TFF3_CRP) and combination of the three markers (TFF3_CRP_FCP) for predicting complete mucosal healing defined as both UCEIS and EMS values of 0. (For abbreviations see Fig. 2).

the predictability of TFF3 and its combination with CRP for evaluating $\mathrm{MH}$ in UC patients.

Conflicts of interest: None to declare. 
Authors' contributions: R.N and V.N designed the study, enrolled and followed up the patients; performed statistical analyses; collected and analyzed the data and wrote the manuscript. T.V. performed the research and participated in study design. V.G. and L.T. managed the patients, collected and analyzed data, and supervised the project. All authors approved the final version of the manuscript

\section{REFERENCES}

1. Magro F, Gionchetti P, Eliakim R, et al. Third European Evidence-based Consensus on Diagnosis and Management of Ulcerative Colitis. Part 1: Definitions, Diagnosis, Extra-intestinal Manifestations, Pregnancy, Cancer Surveillance, Surgery, and Ileo-anal Pouch Disorders. J Crohns Colitis 2017;11:649-670. doi:10.1093/ecco-jcc/jjx008

2. Harbord M, Eliakim R, Bettenworth D, et al. Third European Evidencebased Consensus on Diagnosis and Management of Ulcerative Colitis. Part 2: Current Management. J Crohns Colitis 2017;11:769-784. doi:10.1093/ecco-jcc/jjx009

3. Turner D, Walsh CM, Steinhart AH, Griffiths AM. Response to corticosteroids in severe ulcerative colitis: a systematic review of the literature and a meta-regression. Clin Gastroenterol Hepatol 2007;5:103-110. doi:10.1016/j.cgh.2006.09.033

4. Lichtenstein GR, Rutgeerts P. Importance of mucosal healing in ulcerative colitis. Inflamm Bowel Dis 2010;16:338-346. doi:10.1002/ibd.20997

5. Shah SC, Colombel JF, Sands BE, Narula N. Mucosal healing is associated with improved long-term outcomes of patients with ulcerative colitis: a systematic review and meta-analysis. Clin Gastroenterol Hepatol 2016;14:1245-1255. doi:10.1016/j.cgh.2016.01.015

6. Peyrin-Biroulet L, Sandborn W, Sands BE, et al. Selecting therapeutic targets in inflammatory bowel disease (STRIDE): determining therapeutic goals for treat-to-target. Am J Gastroenterol 2015;110:13241338. doi:10.1038/ajg.2015.233

7. Nikolaus S, Schreiber S. Diagnostics of inflammatory bowel disease Gastroenterology 2007;133:1670-1689. doi:10.1053/j.gastro.2007.09.001

8. Yoon JY, Park SJ, Hong SP, Kim TI, Kim WH, Cheon JH. Correlations of C-reactive protein levels and erythrocyte sedimentation rates with endoscopic activity indices in patients with ulcerative colitis. Dig Dis Sci 2014;59:829-837. doi:10.1007/s10620-013-2907-3

9. Magro F, Lopes SI, Lopes J, et al. Histological outcomes and predictive value of faecal markers in moderately to severely active ulcerative colitis patients receiving infliximab. J Crohns Colitis 2016;10:1407-1416. doi:10.1093/ecco-jcc/jjw112

10. Magro F, Lopes S, Coelho R, et al. Accuracy of faecal calprotectin and neutrophil gelatinase B-associated lipocalin in evaluating sub-clinical inflammation in ulcerative colitis-the ACERTIVE study. J Crohns Colitis 2017;11:435-444. doi:10.1093/ecco-jcc/jjw170

11. Podolsky DK, Lynch-Devaney K, Stow JL, et al. Identification of human intestinal trefoil factor. Goblet cell-specific expression of a peptide targeted for apical secretion. J Biol Chem 1993;268:6694-6702.

12. Aamann L, Vestergaard EM, Gronbaek H. Trefoil factors in inflammatory bowel disease. World J Gastroenterol 2014;20:3223-3230 doi:10.3748/wjg.v20.i12.3223

13. Wright NA, Poulsom R, Stamp G, et al. Trefoil peptide gene expression in gastrointestinal epithelial cells in inflammatory bowel disease. Scand J Gastroenterol 1992;193:76-82.
14. Shaoul R, Okada Y, Cutz E, Marcon MA. Colonic expression of MUC2, MUC5AC, and TFF1 in inflammatory bowel disease in children. J Pediatr Gastroenterol Nutr 2004;38:488-493.

15. Nakov R, Velikova T, Nakov V, Ianiro G, Gerova V, Tankova L. Serum trefoil factor 3 predicts disease activity in patients with ulcerative colitis. Eur Rev Med Pharmacol Sci 2019;23:788-794. doi:10.26355/eurrev_201901_16893

16. Srivastava S, Kedia S, Kumar S, et al. Serum human trefoil factor 3 is a biomarker for mucosal healing in ulcerative colitis patients with minimal disease activity. J Crohns Colitis 2015;9:575-579. doi:10.1093/ ecco-jcc/jjv075

17. Lichtiger S, Present DH, Kornbluth A, et al. Cyclosporine in severe ulcerative colitis refractory to steroid therapy. N Engl J Med 1994;330:1841-1845. doi:10.1056/NEJM199406303302601

18. Travis SP, Schnell D, Krzeski P, et al. Developing an instrument to assess the endoscopic severity of ulcerative colitis: the Ulcerative Colitis Endoscopic Index of Severity (UCEIS). Gut 2012;61:535-542. doi:10.1136/gutjnl-2011-300486

19. Quantum Blue ${ }^{\circ}$ Calprotectin Normal Range Quantitative Lateral Flow Assay LF-CAL20 package insert (2010-11-10).

20. Meng XL, Rosenthal R, Rubin DB. Comparing correlated correlation coefficients. Psychol Bull 1992;111:172-175. doi:10.1037/00332909.111.1.172

21. DeLong ER, DeLong DM, Clarke-Pearson DL. Comparing the areas under two or more correlated receiver operating characteristic curves: a nonparametric approach. Biometrics 1988;44:837-845.

22. Dave M, Loftus EV. Mucosal healing in Inflammatory Bowel Disease - a true paradigm of success? Gastroenterol Hepatol 2012;8:29-38.

23. Vestergaard EM, Poulsen SS, Grønbaek H, et al. Development and evaluation of an ELISA for human trefoil factor 3. Clin Chem 2002;48:1689-1695

24. Grønbaek H, Vestergaard EM, Hey H, Nielsen JN, Nexø E. Serum trefoil factors in patients with inflammatory bowel disease. Digestion 2006;74:33-39. doi:10.1159/000096591

25. Eder P, Stawczyk-Eder K, Korybalska K, et al. Trefoil factor-3 is not a useful marker of mucosal healing in Crohn's disease treated with antiTNF- $\alpha$ antibodies. World J Gastroenterol 2017;23:135-140. doi:10.3748/ wjg.v23.11.135

26. Schoepfer AM, Beglinger C, Straumann A, Trummler M, Renzulli P, Seibold F. Ulcerative colitis: correlation of the Rachmilewitz endoscopic activity index with fecal calprotectin, clinical activity, C-reactive protein, and blood leukocytes. Inflamm Bowel Dis 2009;15:1851-1858. doi:10.1002/ibd.20986

27. Røseth AG, Schmidt PN, Fagerhol MK. Correlation between faecal excretion of indium-111-labelled granulocytes and calprotectin, a granulocyte marker protein, in patients with inflammatory bowel disease. Scand J Gastroenterol 1999;34:50-54.

28. Røseth AG, Fagerhol MK, Aadland E, Schjönsby H. Assessment of the neutrophil dominating protein calprotectin in feces. A methodologic study. Scand J Gastroenterol 1992;27:793-798.

29. Lasson A, Stotzer PO, Ohman L, Isaksson S, Sapnara M, Strid H. The intra-individual variability of faecal calprotectin: a prospective study in patients with active ulcerative colitis. J Crohns Colitis 2015;9:26-32. doi:10.1016/j.crohns.2014.06.002

30. Rokkas T, Portincasa P, Koutroubakis IE. Fecal calprotectin in assessing inflammatory bowel disease endoscopic activity: a diagnostic accuracy meta-analysis. J Gastrointestin Liver Dis 2018;27:299-306. doi:10.15403/ jgld.2014.1121.273.pti 